Anneliese Nusmeier

Johannes G. van der Hoeven

Joris Lemson

\section{Interpretation of the transpulmonary thermodilution curve in the presence of a left-to-right shunt}

Accepted: 1 September 2010

Published online: 9 December 2010 (c) The Author(s) 2010. This article is published with open access at Springerlink.com

An author's reply to this comment is available at doi:10.1007/s00134-010-2108-x.

Dear Editor,

In a recent article by Giraud et al. [1] the effect of a left-to-right shunt on the transpulmonary thermodilution (TPTD) curve and the subsequent calculation of extravascular lung water (EVLW) and intrathoracic blood volume (ITBV) are described. The authors conclude that a left-toright shunt generates recirculation of thermal indicator, which induces a

Table 1 Recalculations for case 1

\begin{tabular}{lll}
\hline Case 1 & VSD present & VSD closed \\
\hline CO & 4.79 & 5.68 \\
ITBVI & 857 & 1,334 \\
EVLWI & 31.7 & 14.3 \\
BSA & 1.8 & 1.8 \\
Weight & 75 & 75 \\
Calculations & & \\
ITBV (ml) & $1,542.6$ & $2,401.2$ \\
EVLW (ml) & $2,377.5$ & $1,072.5$ \\
GEDV (ml) & $1,234.1$ & $1,921.0$ \\
ITTV (ml) & $3,920.1$ & $3,473.7$ \\
MTt (s) & 49.1 & 36.7 \\
PTV (ml) & $2,686.0$ & $1,552.7$ \\
DSt (s) & 33.6 & 16.4 \\
\hline
\end{tabular}

$\mathrm{ITTV}=\mathrm{CO} \times \mathrm{MTt} \times 1,000 / 60$

$\mathrm{PTV}=\mathrm{CO} \times$ DSt $\times 1,000 / 60$

GEDV $=$ ITTV - PTV

$\mathrm{ITBV}=\mathrm{GEDV} \times 1.25$

$\mathrm{EVLW}=\mathrm{ITTV}-\mathrm{ITBV}$ change in the dilution curve.

Although we support their observation their explanation may lead to confusion.

The transpulmonary thermodilution method must fulfill the following conditions: (1) constant blood flow, (2) no or minimal loss of indicator between injection and detection point, (3) complete mixing of the indicator with blood, and (4) the indicator must pass the detection point only once [2]. To satisfy condition 4 , the dilution curve is interrupted at the downslope part based upon a specific algorithm to prevent the effects of recirculation [3]. Subsequently the curve is extrapolated from the interrupted point to the baseline in order to calculate the area under the curve [4].

The cases described by Giraud et al. both had a left-to-right shunt (ventricular septal defect (VSD) and aorto-cava fistula (ACF)). The (pulmonary) "recirculation" that occurs "short" circuit. The observed TPTD curve is the result of a delay in delivery of the indicator to the systemic circulation and will subsequently show a lower initial peak, followed by a slow re-approximation to the baseline. in a left-to-right shunt is an extra
Unfortunately the authors do not provide the values of the mean transit time (MTt) and downslope time (DSt), but these numbers are easily deduced from Fig. 1 on page 1,084 [1]. The recalculations are explained in Table 1 and show that the left-toright shunt induces an increase of both time intervals. The increase of the DSt $(51 \%)$ is twice the increment of the MTt (25\%). Recirculation of the indicator passing the detecting point for a second time is excluded by the fact that true recirculation will not occur before approximately $60 \mathrm{~s}$ ( $\approx 2 \times$ MTt in the normal situation). This is long after the interruption of the downslope part.

Increment of the DSt and, to a lesser extent, of the MTt can also be observed in the presence of a large volume of lung water. Both situations are the consequence of delayed delivery of indicator to the systemic circulation. In conclusion it can be stated that a left-to-right shunt induces an increase in DSt and, to a lesser extent, MTt as a consequence of delayed delivery of indicator to the systemic circulation because of the presence of an extra circuit. This phenomenon should not be confused with true recirculation.

Conflict of interest The authors have not disclosed any potential conflicts of interest.

Open Access This article is distributed under the terms of the Creative Commons Attribution Noncommercial License which permits any noncommercial use, distribution, and reproduction in any medium, provided the original author(s) and source are credited.

\section{References}

1. Giraud R, Siegenthaler N, Park C, Beutler S, Bendjelid K (2010)

Transpulmonary thermodilution curves for detection of shunt. Intensive Care Med 36:1083-1086. doi:

10.1007/s00134-010-1876-7 
2. Zierler KL (1962) Theoretical basis of indicator-dilution methods for measuring flow and volume. Circ Res 10:393-407

3. Nadeau S, Noble WH (1986) Limitations of cardiac output measurements by thermodilution. Can Anaesth Soc J 33:780-784
4. Reuter DA, Huang C, Edrich T, Shernan SK, Eltzschig HK (2010) Cardiac output monitoring using indicator-dilution techniques: basics, limits, and perspectives. Anesth Analg 110:799-811
A. Nusmeier $(\bowtie)$ J. G. van der Hoeven • J. Lemson

Department of Intensive Care Medicine, Radboud University Nijmegen Medical Centre, Internal postal code 632,

PO Box 9101, 6500 HB Nijmegen,

The Netherlands

e-mail: a.nusmeier@ic.umcn.nl

Tel.: +31-24-3617273

Fax: +31-24-3541612 